\title{
Explicitation du savoir professionnel novice en Santé, assistance et soins infirmiers : un processus de raisonnement de métier
}

Auteures

Ghyslaine Daigle, M. Éd. agente de recherche Université du Québec à Rimouski, Canada, Ghyslaine_Daigle@uqar.ca

Marie Alexandre Ph. D., Professeure en psychopédagogie de l'enseignement professionnel, Université du Québec à Rimouski, Canada, marie alexandre@ugar.ca 


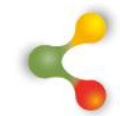

\section{REVUE HYBRIDE DE L'ÉDUCATION}

\section{Résumé}

L'élargissement du champ d'exercices défini par l'Ordre des infirmiers et infirmières auxiliaires $d u$ Québec impose une hausse du niveau de compétences attendues, notamment en ce qui concerne le degré d'autonomie et le maintien de conditions d'exercice sécuritaires. Les écarts entre les savoirs de la formation et les savoirs requis par le marché du travail semblent préoccupants. Cet article présente des résultats d'une étude sur le savoir professionnel infirmier novice fondé sur le processus de raisonnement de métier. Les résultats d'un entretien d'explicitation conduit auprès d'une personne novice en première année de pratique suggèrent l'exercice d'un processus de résolution de problème soutenant l'agir professionnel infirmier.

Mots-clés: analyse de l'activité; didactique professionnelle; entretien d'explicitation; processus de raisonnement de métier; professionnalisation; savoir professionnel 


\section{$\&$}

\section{REVUE HYBRIDE DE L'ÉDUCATION}

\section{Problématique}

\subsection{De nouvelles compétences professionnelles}

Au cours de la dernière décennie, la pratique des infirmiers et des infirmières auxiliaires s'est complexifiée avec l'ajout de nombreux actes autorisés (gouvernement du Québec, 2003; Ordre des Infirmières et Infirmiers auxiliaires du Québec, 2014). Ces modifications majeures impliquent une hausse significative des compétences requises pour l'exercice de la profession, tout en maintenant un degré d'autonomie nécessaire au maintien de conditions d'exercice sécuritaires (Fédération interprofessionnelle de la Santé du Québec, 2009). Cette transformation du rôle professionnel suscite des questionnements quant à la formation permettant d'assurer adéquatement l'ensemble des responsabilités à l'arrivée sur le marché du travail (Direction des soins infirmiers, 2009 ; fédération interprofessionnelle de la santé du Québec, 2009 ; Hould, 2011). Or, les écarts entre les savoirs de la formation et les savoirs requis par le marché du travail sont préoccupants. Les personnes nouvellement diplômées rencontrent des difficultés pour répondre aux exigences de plus en plus complexes du marché du travail. En effet, ce rôle élargi entraîne l'accroissement de responsabilités et nécessite une capacité d'autorégulation qui va bien au-delà du rôle d'assistante qui leur était autrefois dévolu. Considérant que le savoir professionnel correspond à un processus décisionnel mobilisant des stratégies d'action (Hardy et Landreville, 1992 ; Pastré, 2002 ; Perrenoud, 2004 ; Roegiers, 2010), quel est le savoir infirmier détenu et mis en pratique par des personnes infirmières auxiliaires nouvellement diplômées de la formation professionnelle en première année de pratique?

\subsection{De garde-malade à infirmier et infirmière auxiliaire : une évolution constante}

Apparu au milieu du XXe siècle afin de combler un manque de personnel soignant, le métier d'infirmier et d'infirmière auxiliaire est en constante évolution. Au départ, ces personnes étaient vouées à l'entretien domestique de la chambre et aux soins de base des malades. Toutefois, à la suite de longues années de négociations, l'article 36 de la Loi sur les infirmiers et infirmières (gouvernement du Québec, 2003) autorise 16 actes infirmiers supplémentaires. En outre, l'article 31 de la Loi médicale (gouvernement du Québec, 2019a) ajoute 43 actes médicaux à la pratique. 


\section{REVUE HYBRIDE DE L'ÉDUCATION}

Tableau 1 : Évolution de la profession de 1923 à 2008

\begin{tabular}{l|l}
\hline 1923-1970 & $\begin{array}{l}\text { De la première école privée de "gardes-malades » } \\
\text { auxiliaires à la disparition des écoles dans les hôpitaux } \\
\text { Intégration au ministère de l'Éducation du Québec (MEQ). }\end{array}$ \\
\hline $\mathbf{1 9 8 0 - 1 9 9 0}$ & $\begin{array}{l}\text { Autorisation de 16 actes infirmiers (Loi sur les infirmières et } \\
\text { infirmiers, art. 36). Autorisation à contribuer et pratiquer 43 } \\
\text { actes médicaux (Loi médicale, art. 31). }\end{array}$ \\
\hline $\mathbf{1 9 9 0 - 1 9 9 4}$ & $\begin{array}{l}\text { Implantation du programme de formation par le MEQ et la } \\
\text { CllA } \\
\text { Création de l'Ordre des Infirmières et Infirmiers auxiliaires } \\
\text { du Québec }\end{array}$ \\
\hline 2003-2008 & Autorisation de 11 nouveaux actes délégués (OIIAQ, 2014). \\
\hline
\end{tabular}

Par ailleurs, le champ d'exercice de plusieurs professions dans le domaine de la santé s'est considérablement modifié au cours de la dernière décennie avec l'entrée en vigueur de la Loi 90 modifiant le Code des professions (gouvernement du Québec, 2003). En effet, en 2003, la Loi 90 donne plus de latitude aux pratiques des infirmiers et des infirmières auxiliaires avec la délégation de neuf activités réservées. Ces activités sont dites réservées «[...] en raison du risque de préjudice lié à leur réalisation ainsi que des compétences requises et des connaissances exigées pour les exercer » (fédération interprofessionnelle de la Santé du Québec, 2009, p. 5). 


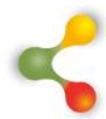

REVUE HYBRIDE DE L'ÉDUCATION

\section{Tableau 2 : Activités déléguées par la Loi modifiant le Code des professions (Loi 90)}

\begin{tabular}{l|l}
\hline $\begin{array}{l}\text { Mélanger des substances en vue } \\
\text { de compléter la préparation d'un } \\
\text { médicament selon une ordonnance }\end{array}$ & $\begin{array}{l}\text { Administrer, par des voies autres que la voie } \\
\text { intraveineuse, des médicaments ou d'autres } \\
\text { substances, lorsqu'ils font l'objet d'une } \\
\text { ordonnance }\end{array}$ \\
\hline $\begin{array}{l}\text { Effectuer des prélèvements selon } \\
\text { une ordonnance }\end{array}$ & $\begin{array}{l}\text { Introduire un instrument, selon une } \\
\text { ordonnance, dans une veine périphérique à } \\
\text { des fins de prélèvement }\end{array}$ \\
\hline $\begin{array}{l}\text { Appliquer les mesures invasives } \\
\text { d'entretien du matériel théra- } \\
\text { peutique }\end{array}$ & $\begin{array}{l}\text { Contribuer à la vaccination dans le cadre } \\
\text { d'une activité découlant de l'application de la } \\
\text { Loi sur la santé publique }\end{array}$ \\
\hline $\begin{array}{l}\text { Observer l'état de conscience d'une } \\
\text { personne et surveiller les signes } \\
\text { neurologiques }\end{array}$ & $\begin{array}{l}\text { Prodiguer des soins et des traitements reliés } \\
\text { aux plaies et aux altérations de la peau et des } \\
\text { téguments, selon une ordonnance ou selon } \\
\text { le plan de traitement infirmier }\end{array}$ \\
\hline
\end{tabular}

Introduire un instrument ou un doigt, selon une ordonnance, au-delà du vestibule nasal, au-delà des grandes lèvres, du méat urinaire ou de la marge de l'anus ou dans une ouverture artificielle du corps humain

(adapté de OIIAQ, 2011, p. 10-14)

Plus spécifiquement, l'élargissement du rôle professionnel de l'infirmier et de l'infirmière auxiliaire se traduit par la complexification de tâches exigeant des capacités d'adaptation et de discernement qui vont bien au-delà de l'exécution de gestes techniques traditionnellement relégués à une aide-soignante (fédération interprofessionnelle de la santé du Québec, 2008). En augmentant leur implication dans tous les domaines de soins, les personnes infirmières auxiliaires portent de nouvelles responsabilités, tant sociales que professionnelles. Leur champ d'exercices est ainsi défini par l'Ordre des infirmiers et infirmières auxiliaires du Québec (2011) :

[...] contribuer à l'évaluation de l'état de santé d'une personne et à la réalisation du plan de soins, prodiguer des soins et des traitements infirmiers et médicaux dans le but de maintenir la santé, de la rétablir, de prévenir la maladie et fournir des soins palliatifs (p. 5).

Or, au regard du programme de formation dispensé dans les centres de formation professionnelle, aucune modification quant au nombre d'heures d'apprentissage nécessaires à l'acquisition des nouvelles compétences n'a été apportée (MELS, 2009 ; MEQ, 2000). De 1800 heures à l'entrée en vigueur de la loi, le programme "Santé, assistance et soins infirmiers (SASI) » totalise le même nombre d'heures aujourd'hui, et ce, malgré l'ajout significatif d'actes professionnels spécifiques. Les nouvelles 


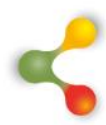

\section{REVUE HYBRIDE DE L'ÉDUCATION}

connaissances à acquérir sont insérées à l'intérieur des blocs de compétences déjà enseignées (MELS, 2009).

Depuis 2003, date d'entrée en vigueur du règlement, l'Ordre des infirmiers et infirmières auxiliaires du Québec impose à ses membres en place au sein du réseau un nombre d'heures de mise à jour. En effet, le règlement sur les activités de formation continue des infirmiers et infirmières auxiliaires en détermine les balises. Ces activités de mise à niveau sont obligatoires à la poursuite des activités professionnelles et doivent être réalisées dans un délai de 18 mois (gouvernement du Québec, 2003, p. 2323-2324).

Tableau 3 : Mises à jour activités réservées

\begin{tabular}{|l|l|}
\hline Activité & Exigences \\
\hline $\begin{array}{l}\text { Prélèvement sanguin par } \\
\text { ponction veineuse }\end{array}$ & $\begin{array}{l}\text { Formation théorique : au moins } 5 \text { heures } \\
\text { Formation pratique : au moins 5 heures }\end{array}$ \\
\hline Administration des vaccins & $\begin{array}{l}\text { Formation théorique : au moins 4 heures } \\
\text { Formation pratique : au moins 2 heures }\end{array}$ \\
\hline $\begin{array}{l}\text { Installation du tube naso- } \\
\text { gastrique }\end{array}$ & $\begin{array}{l}\text { Formation théorique : au moins 2 heures } \\
\text { Formation pratique : au moins 2 heures }\end{array}$ \\
\hline
\end{tabular}

Des exigences de formation supplémentaires pour le personnel en place sont à nouveau ajoutées en 2008 par l'Ordre des infirmiers et infirmières auxiliaires du Québec, à la suite de l'autorisation d'exercer deux nouvelles activités professionnelles, dites «autorisées », c'est-à-dire qu'elles sont soumises à certaines conditions, dont la présence d'un plan thérapeutique infirmier. Dans certains cas, la personne infirmière doit être disponible en vue d'une intervention rapide si cela est nécessaire (OIIAQ, 2011, p. 15-17). 


\section{$\&$}

REVUE HYBRIDE DE L'ÉDUCATION

Tableau 4 : Mises à jour activités autorisées

\begin{tabular}{|l|l}
\hline Activité & Exigences \\
\hline $\begin{array}{l}\text { Entretien d'une trachéostomie } \\
\text { reliée à un ventilateur }\end{array}$ & $\left.\begin{array}{l}\text { Formation théorique } \\
\text { Formation pratique }\end{array}\right\}$ au moins 7 heures \\
$\begin{array}{l}\text { Contribution à la thérapie } \\
\text { intraveineuse }\end{array}$ & $\left.\begin{array}{l}\text { Formation théorique } \\
\text { Formation pratique }\end{array}\right\}$ au moins 21 heures
\end{tabular}

\subsection{Complexification et professionnalisation du rôle infirmier en Santé, assistance et soins infirmiers}

La notion de professionnalisme prévoit que «l'exercice d'une profession implique une activité intellectuelle qui engage la responsabilité individuelle de celui qui l'exerce» (Perrenoud, 1994, p. 3). La pratique en Santé, assistance et soins infirmiers implique un engagement personnel dans la gestion des situations complexes, l'analyse et le choix de moyens d'intervention adaptés à la situation rencontrée. II ne s'agit plus simplement d'exécuter des consignes, mais de choisir et d'appliquer les actes adéquats, dans une situation donnée, en tenant compte de critères d'éthique, de déontologie et de sécurité. De ce fait, la pratique d'infirmier et d'infirmière auxiliaire rejoint le concept de professionnalité défini comme "le développement d'un savoir d'expérience ou à la jonction entre des savoirs et une situation » (Perrenoud, 2004, p. 27). Roegiers (2010) précise que la professionnalisation.

[...] va donc plus loin qu'une exécution technique de tâches : elle englobe la capacité à pouvoir réagir face à l'imprévu ; elle englobe aussi l'analyse régulière des situations de travail et les régulations que le professionnel introduit suite à son analyse (p. 27).

La personne professionnelle n'est pas qu'une exécutante. Elle doit prendre des décisions et s'adapter constamment aux changements qui s'opèrent autour d'elle. De plus, il lui faut tenir compte du fait que toutes les actions peuvent à un moment ou à un autre être influencées par de nombreux facteurs, qu'ils soient individuels, contextuels ou encore organisationnels. À cet égard, selon Perrenoud (1994), la dimension professionnelle se reconnaît à la capacité d'établir un rapport pragmatique aux savoirs théoriques. Plus précisément, pour cet auteur, il s'agit de mettre de l'avant des capacités d'action face à une situation complexe. En effet, la complexité, la diversité, la mobilité des situations de soins et des décisions arriment étroitement la conception et l'exécution de l'action. 


\section{$\&$}

\section{REVUE HYBRIDE DE L'ÉDUCATION}

Par situation de soins, on entend toute situation au cours de laquelle une personne soignante entre en relation avec une personne soignée, lui porte une attention soutenue et lui prodigue des soins d'assistance ou des soins et des traitements infirmiers et médicaux dans un environnement précis. Toutefois, les tâches d'évaluation et d'intervention propres à toute situation de soins sont de la responsabilité de la personne infirmière possédant au moins un diplôme d'études collégiales (licenciée) dans son rôle « d'évaluer l'état de santé d'une personne »(OIIAQ, 2011, p. 32).

Pourtant, dans la pratique, il ressort que l'infirmier et l'infirmière auxiliaire adoptent également la position de juge préalable à l'intervention, pour être en mesure d'évaluer et de décider de la meilleure solution dans une situation donnée. La capacité de jugement est maintenant essentielle. En effet, I'OIIAQ (2011), dans un document balisant les activités professionnelles de ses membres, précise, en parlant de l'activité visant à surveiller et à maintenir une perfusion intraveineuse, que la personne « [...] infirmière auxiliaire assume cette responsabilité pour toutes les solutions intraveineuses qu'elle peut administrer, dans le respect de l'ordonnance et de la directive infirmière lorsque indiquée » (p. 36).

Le personnel infirmier fait face à un dilemme particulier alors que la pratique diffère des théories formelles. À ce propos, une étude de Benner (1995), menée auprès de 42 infirmières dans trois hôpitaux, constate que "si on oblige les expertes à faire attention à des détails, à un modèle ou à une règle formelle, leurs performances se détériorent » $(p .36)$.

Benner (1995) fait valoir le rôle majeur de l'expérience dans l'évaluation d'une situation de soins. Le jugement infirmier passe par la conscience perceptive, d'abord traduite par de vagues intuitions en dehors de l'analyse. Ces pressentiments guident l'action première des personnes infirmières expertes qui, par la suite, vérifient leurs perceptions pour parfaire l'évaluation de l'état du malade. Benner (1995) soutient que la confrontation à des situations réelles par une remise en question des notions établies est nécessaire au développement de l'expertise. Les résultats d'une étude menée par Lechasseur (2009) auprès d'étudiantes en sciences infirmières sur la pensée critique révèlent un type de savoir qui repose sur des perceptions, des sensations corporelles ou encore de vagues impressions. Le savoir perceptuel correspond à «une compréhension des signes subtils chez la personne, permettant de mieux la connaître et de mieux saisir l'évolution de sa condition clinique " (Lechasseur, 2009, p.267).

L'expérience correspond à « l'amélioration de théories et de notions préconçues au travers de la rencontre de nombreuses situations réelles qui ajoutent des nuances ou des différences subtiles à la théorie " (Lechasseur, 2009, p.36). La vision de «ce qui est possible » est l'une des 


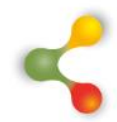

\section{REVUE HYBRIDE DE L'ÉDUCATION}

caractéristiques de l'expertise traduite par l'utilisation d'un processus analytique qui contribue à transformer la compétence et conduit de ce fait à l'amélioration des performances. La compétence peut alors correspondre à l'établissement des liens entre la complexité du milieu de soins et les théories formelles. La mobilisation d'un processus décisionnel en situation de soins requiert la réalité de la pratique (Benner,1995). Pastré (2016) souligne que «l'action dans le travail » contribue au développement des compétences. De même, Roegiers (2012) affirme que les savoirs de métier ne se manifestent qu'en situation réelle.

L'activité professionnelle doit être envisagée en contexte. L'efficacité professionnelle requiert la capacité à combiner et à transformer des contenus "en une série de savoir-faire liés aux tâches du métier " (Roegiers, 2010, p.18). À la suite de leurs travaux sur les savoirs professionnels, Hardy et Landreville (1992) considèrent que ces derniers doivent être observés par rapport à leur contenu, mais en plus, au regard du rôle social perçu par l'élève en formation.

\subsection{Les nouvelles personnes diplômées sur le marché du travail : une difficile adaptation}

La difficulté des nouvelles personnes diplômées à s'adapter au milieu du travail en soins infirmiers est observée dans plusieurs centres hospitaliers. Les directions d'établissements éprouvent le besoin de parfaire la formation des nouvelles personnes embauchées (Direction des soins infirmiers, 2009; Hould, 2011). En outre, les directions d'établissements considèrent essentielle l'instauration de programmes visant l'intégration professionnelle en favorisant le développement de nouvelles connaissances, mais aussi la pratique de soins de qualité de façon sécuritaire (Direction des soins infirmiers, 2009).

En somme, l'élargissement de la pratique professionnelle impose une hausse des compétences requises en Santé, assistance et soins infirmiers notamment au regard du degré d'autonomie et du maintien de conditions d'exercice sécuritaires. Or, face à la complexification et la professionnalisation du rôle d'infirmier et d'infirmière auxiliaire, l'écart entre les savoirs de la formation et les savoirs requis par le marché du travail ne cesse de se creuser. En outre, le développement des compétences professionnelles prend appui sur un arrimage entre la théorie et la pratique afin de faciliter l'adaptation des nouvelles personnes diplômées sur le marché du travail.

Cet article rend compte d'une étude menée sur le savoir professionnel détenu et mis en pratique par une infirmière auxiliaire diplômée du programme "Santé, assistance et soins infirmiers" en première année de pratique. La réduction du décalage entre les 


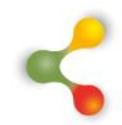

\section{REVUE HYBRIDE DE L'ÉDUCATION}

compétences acquises en formation et celles qui sont requises en milieu de travail, étant le cœur de cette recherche, s'inscrit dans la didactique professionnelle. Le cadre théorique, présenté dans la section suivante, est fondé sur le processus de raisonnement de métier (Alexandre, Thériault et Daigle 2016) permettant de poser un regard innovant sur la pensée de métier.

\section{Cadre théorique}

\subsection{Le savoir infirmier : un enjeu de la didactique professionnelle}

La didactique professionnelle propose de comprendre la nature et les évolutions du travail ainsi que leurs conséquences sur les personnes professionnelles en termes de connaissances et de compétences. Ce champ de recherche examine notamment les conditions et les processus d'apprentissage dans le travail, par le travail et par la formation. En ce sens, la conceptualisation de l'action reconnaît que la personne professionnelle exerce et exprime son intelligence au travail en identifiant et en interprétant les situations avec lesquelles il doit agir (Association de didactique professionnelle, 2018).

Lechasseur (2009) identifie le savoir infirmier comme étant «la compréhension des signes subtils chez la personne, permettant de mieux la connaître et de mieux saisir l'évolution de sa condition clinique » (Lechasseur, 2009, p. 267). Elle identifie dix types de savoirs propres à la profession infirmière. 


\section{REVUE HYBRIDE DE L'ÉDUCATION}

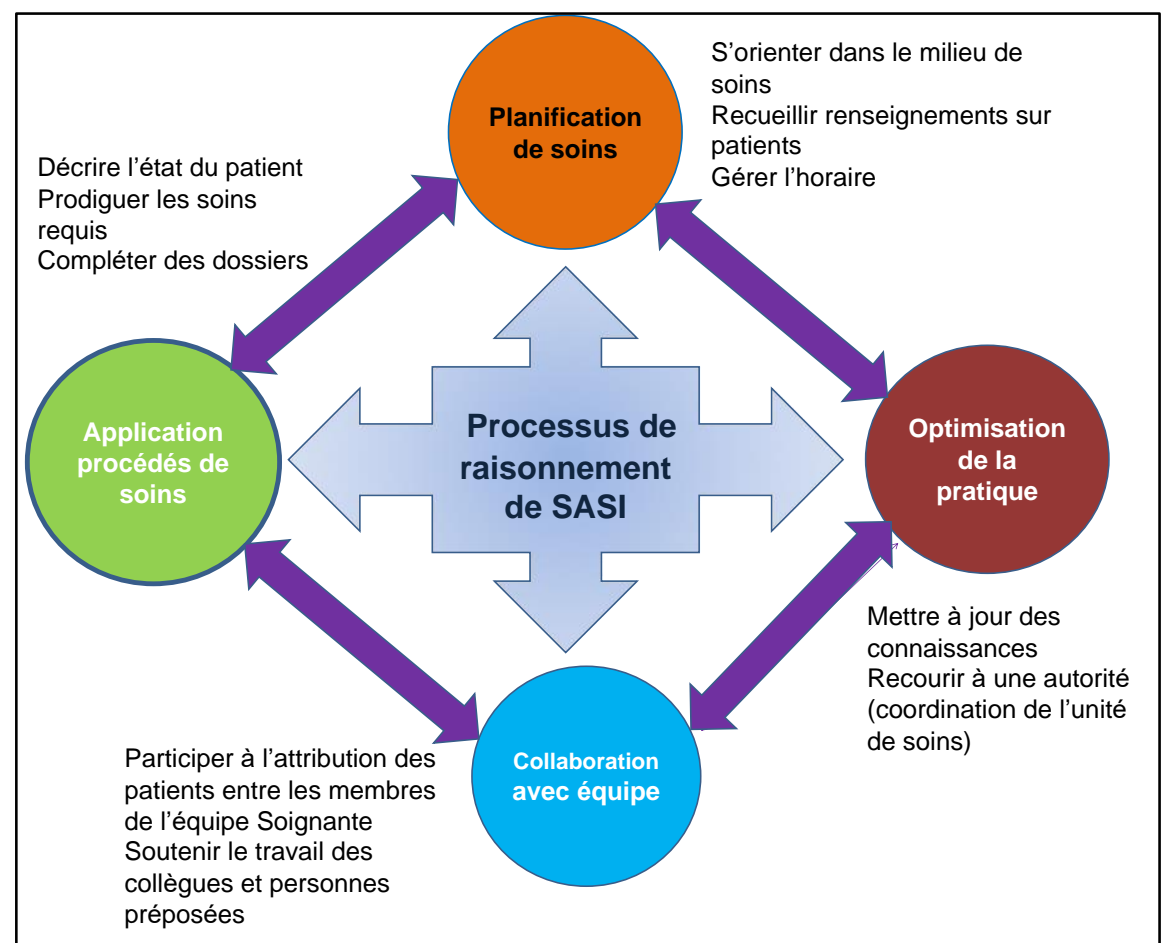

Figure 1 : Activités clés et actions du processus de raisonnement en Santé, assistance, et soins infirmiers (SASI)

(adapté de Lechasseur, 2009)

La notion de fonctionnements créatifs établie par Perrenoud (1994) met en évidence l'utilisation de mécanismes intuitifs pour s'adapter aux situations, jumelée à la nécessité d'utiliser l'ensemble des savoirs pour exercer une compétence. Pour sa part, Roegiers (2010) désigne l'intégration des savoirs comme étant

«[...]la mobilisation conjointe, par l'apprenant, de plusieurs savoirs et savoir-faire, pour résoudre une situation complexe, ou pour réaliser une tâche complexe. C'est chaque apprenant qui intègre, en mobilisant des ressources acquises antérieurement » (p. 201).

L'exercice d'un métier correspond à l'intégration des savoir-faire en situation et des compétences professionnelles. En soins infirmiers, Roegiers (2010) reconnaît quatre activités clés : (1) la réalisation des prestations de soins (2) l'organisation du travail du service (3) la gestion des relations avec le patient et sa famille et (4) le suivi médical et administratif du patient » (p. 22). Roegiers (2010) affirme que ces compétences ne se développent qu'en 


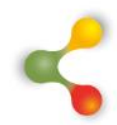

\section{REVUE HYBRIDE DE L'ÉDUCATION}

exerçant le métier et se retrouvent dans des situations plus complexes que les savoir-faire professionnels. Alexandre, Thériault et Daigle (2016), qui se sont intéressées à l'adéquation entre la formation et l'emploi, soulignent que

La réduction de l'écart entre les savoirs de la formation et ceux du marché du travail requiert l'identification de types de savoirs inclus dans les divers programmes de formation ainsi que la compréhension de la nature du savoir de métier (p. 4).

\subsection{L'analyse du travail : pour arrimer la formation et l'emploi}

Depuis ses débuts, la didactique professionnelle accorde une place prépondérante à l'analyse du travail en situation réelle. Issue de deux courants théoriques, la conceptualisation dans l'action, initiée par Vergnaud (1992, dans Pastré 2002) et la psychologie du travail de Leplat (2000), la didactique professionnelle a pour objectifs de construire des contenus de formation répondant au domaine de compétence souhaité et d'utiliser des situations de travail dans le processus d'acquisition des compétences (Pastré, 2002).

La psychologie du travail a permis à la didactique professionnelle de contribuer à l'analyse cognitive des situations de travail en plus d'y apporter des techniques et des méthodes. Ainsi, par le travail, le sujet s'adapte aux situations en effectuant une démarche active, ce qui implique un diagnostic et un processus de résolution de problèmes conduisant à la mise en place de stratégies (Pastré, 2002). En accord avec Leplat (2000), Pastré (2002) différencie le travail réel de la tâche prescrite en tenant compte de la part de créativité et d'adaptation inhérente au travail. L'activité, ne pouvant être planifiée complètement, est toujours plus importante que la tâche demandée. Ainsi, la structure cognitive de la tâche oriente l'activité en fonction de dimensions objectives de la situation, sans se référer uniquement à la prescription. L'analyse du travail par la situation inclut la prescription et l'aspect cognitif de la tâche et de l'activité ainsi que son analyse.

\subsection{Le savoir professionnel : un processus de raisonnement}

Savoyant (1979, dans Pastré, 2002) considère que tout travail implique des opérations d'exécution, de contrôle et d'orientation. Si les deux premières opérations sont facilement analysables, l'orientation, composante cognitive de l'activité professionnelle, est peu visible, mais n'en constitue pas moins le noyau de la compétence. L'orientation contribue à identifier les caractéristiques particulières servant à guider l'action. Ainsi, une orientation incomplète affectera directement les 


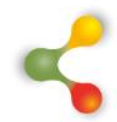

\section{REVUE HYBRIDE DE L'ÉDUCATION}

opérations d'exécution et de contrôle, les opérations d'orientation étant dépendantes de la représentation de la situation que se fait le sujet. L'orientation donne l'importance à la cognition dans l'analyse du travail dans le sens où les éléments organisateurs de l'action sont basés sur les dimensions d'une situation sélectionnée, ce qui constitue le processus du travail (Pastré, 2002).

À son entrée sur le marché du travail, la personne novice possède un savoir morcelé, élaboré à partir des situations vécues. Selon Durand (2005), la personne professionnelle apprend aussi de ses collègues. En effet, le partage d'expériences l'incite à réfléchir à des avenues non envisagées, ce qui vient remplacer le savoir théorique scolaire. La capacité de mettre en lien ces types de savoirs conduit au développement du savoir professionnel qui vise "la résolution d'un problème professionnel n'ayant pas fait l'objet d'un exercice préalable » (Hardy et Landreville, 1992, p.467).

La résolution d'un problème professionnel requiert l'identification adéquate de ses éléments constitutifs et un transfert de l'ensemble de ses connaissances théoriques et pratiques, tout en considérant les particularités disciplinaires. Ceci rejoint la perspective didactique mise de l'avant dans les travaux d'Alexandre (2013) sur le savoir didactique d'enseignantes en technique d'éducation à l'enfance.

Partant du lien entre les représentations des situations et les représentations de l'action, Leplay (2006) considère que «c'est précisément à ce point d'articulation que se situe et que se joue le caractère professionnel de l'action, de la pensée et du savoir » (p. 11). Ce système implique trois types de représentations en interrelations; a) des situations professionnelles, b) des actions associées à ces situations et c) des résultats attendus de ces actions associées à ces situations professionnelles. Ainsi, le travail moderne nécessite la compréhension du fonctionnement des systèmes pour pouvoir y intervenir. En plus d'appliquer des procédures, la personne doit être en mesure de poser un diagnostic et de résoudre des problèmes en tenant compte de leurs dimensions.

La notion de compétence se traduit par le fait qu' « on insiste moins sur l'application des procédures, et plus sur l'intelligence de la tâche et la résolution de problèmes à dimensions multiples " (Pastré, 2016, p. 5). Leplay (2006) précise que le savoir professionnel est l'établissement d'un énoncé de relation entre un système de représentations d'une situation professionnelle et un système de représentations de l'action jumelé à l'expression de la relation par un jugement prédictif, relatif ou absolu, de cohérence ou de pertinence. II peut alors correspondre à un processus de raisonnement de métier, défini selon une forme de "délibération» situationnelle constituée de paliers décisionnels et opérationnels. II soustend un ensemble complexe et hiérarchisé d'activités clés à partir 


\section{$\&$}

\section{REVUE HYBRIDE DE L'ÉDUCATION}

desquelles se déploient des actions en étroite corrélation (Alexandre et al., 2016).

\section{Méthodologie}

Tenant compte du contexte de leur pratique en situation de soins, l'analyse des savoirs détenus et mobilisés par les personnes nouvellement diplômées en première année de pratique a guidé le choix d'une approche compréhensive. La recherche prend appui sur une étude de cas multiples qualitative qui privilégie le point de vue des acteurs (Paillé et Mucchielli, 2003). L'analyse particulière de chaque cas ainsi que la capacité d'en découvrir les convergences apparaissent comme le choix le plus approprié pour cette étude (Karsenti et Demers, 2011). Une grande place est accordée à la vision élargie d'une situation et à la possibilité d'établir une proximité avec les personnes participantes. Les observations, effectuées dans un environnement naturel, permettent d'établir un portrait du sujet en donnant beaucoup d'informations sur l'ensemble de la situation (Roy, 2009).

\subsection{Déroulement de la recherche}

Les cas ont été sélectionnés de façon non aléatoire, afin d'en tirer une description précise et une interprétation qui dépasse ses bornes (Roy, 2009 , p. 207). Quatre personnes nouvellement diplômées ont été recrutées à l'aide d'enseignantes du programme en Santé, assistance et soins infirmiers dans différentes régions du Québec. Les personnes participantes sont quatre femmes détenant un diplôme d'études professionnelles en Santé, assistance et soins infirmiers qui exercent dans un milieu de soins depuis moins d'un an.

La technique de collecte de données est l'entretien d'explicitation qui met en relief la capacité des personnes à faire acte de conscience pour réfléchir au vécu. Les entretiens ont été menés selon les caractéristiques de la technique d'explicitation développée par Vermersch (2014) : le vécu de l'action et le maintien dans un moment spécifié.

Lors de la démarche d'analyse, les étapes du modèle de la sémiose (Vermersch, 2012) ont été appliquées aux données. "La sémiose naît de la distinction et de la mise en relation entre un "objet", une chose à laquelle on peut se référer, donc un référent, et son "représentant", pour un sujet " (Vermersch, 2012, p. 333). Le concept de reprise, organisé autour de l'apparition du couple référent (vécu de référence)/représentant, a permis d'investiguer les créations de sens (Vermersch, 2012). D'abord, tous les entretiens ont été transcrits. Les différents niveaux de reprises articulés autour d'un référent devenu représentant donnent progressivement sens 


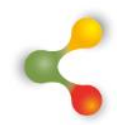

\section{REVUE HYBRIDE DE L'ÉDUCATION}

aux entretiens d'explicitation. Ainsi, de la première unité sémique ( $v .1)$ aux diverses reprises constituant la synthèse progressive du traitement, de l'organisation et de l'analyse des données (rep.9), l'utilisation synergique du couple référent/représentant met en évidence des résultats permettant de modéliser l'exercice du processus soutenant l'agir professionnel. L'analyse suggère que les infirmiers et les infirmières auxiliaires exercent un processus de raisonnement solidement appuyé sur un réseau d'activités clés et d'actions.

Plusieurs mesures en conformité avec les principes énoncés dans la politique institutionnelle en matière d'éthique de la recherche avec les êtres humains de l'Université du Québec à Rimouski ont été prises. Avant le début de la recherche, le consentement écrit de la participante a été obtenu. Elle a été informée du fait qu'elle pouvait en tout temps refuser de participer ou se retirer de la recherche. Par la suite, elle a reçu la garantie de la confidentialité des données recueillies et de la préservation de l'anonymat. La prochaine section présente les résultats de Sara. Cette participante est la seule à posséder de l'expérience de travail dans plus d'un milieu de soins.

Les résultats présentés dans la prochaine section tendent à montrer des capacités de prise d'informations, d'inférences pertinentes ainsi que la mise à jour d'un répertoire d'actions en réponse à la complexité de la situation de travail. Le recours au modèle de la sémiose (Vermersch, 2012) dans le cas de Sara a permis de documenter les actions matérielles, matérialisées et mentales grâce aux différents paliers de reprises qui en constituent l'intelligence organisatrice.

\section{Présentation des résultats : le processus de raisonnement}

L'analyse des résultats de Sara suggère l'exercice d'un processus de travail structuré selon des activités clés et des actions. Plus particulièrement, le processus de raisonnement en Santé, assistance et soins infirmiers comprend des actions associées à quatre activités clés : la planification des soins en fonction du milieu, l'application des procédés de soins, la collaboration avec l'équipe de soins et l'optimisation de la pratique.

\subsection{Actions de l'activité clé Planification des soins en fonction du milieu}

Lors de l'activité clé de planification des soins en fonction du milieu, Sara s'oriente dans le milieu de soins, recueille les renseignements sur les patients et gère son horaire. 


\section{8}

\section{REVUE HYBRIDE DE L'ÉDUCATION}

J'organise ma planification (N26:80) (...). Je sais ma routine, je sais ce que j'ai à faire (N154:452) (Sara).

L'action de s'orienter dans le milieu de soins revêt une importance primordiale. Sara a reçu des orientations dans trois milieux de soins différents (hôpital, chirurgie). De plus, en CHSLD, certaines des routines apprises lors de sa première orientation, qui était dans le système de santé québécois ont déjà changé. Cela réfère à un processus particulier d'initiation à un nouveau cadre de travail. Sara se prépare à ses interventions en recueillant des renseignements sur les patients. Lorsqu'il y a de nouveaux patients, elle ne sait pas à quoi s'attendre. L'action de recueillir les renseignements sur les patients comprend la reconnaissance des cas lourds, la rédaction des dossiers, les pansements à refaire, les médicaments à donner incluant le soulagement des douleurs avec la prise de narcotiques et la pose de blocs salins.

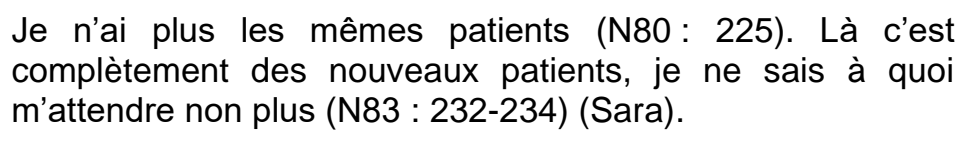

Sara apporte son aide aux préposés concernant les toilettes du matin, les repas ou encore les couchers. L'action de gérer l'horaire permet d'anticiper les événements.

\subsection{Actions de l'activité clé application des procédés de soins}

L'activité clé application des procédés de soins comprend l'ensemble des techniques et des procédures et se déploie selon trois actions distinctes. Lors de l'action de repérer l'état du patient, Sara constate l'état de fatigue du patient et la présence d'un soluté sur l'autre bras.

Je me sens insécure parce que je sais qu'il n'y a pas de veines (N115: 338-339). Je voyais... je sentais un peu la veine. Je ne veux pas prendre tout le bras et manquer une autre fois (N123: 357). Je trouve difficile de prendre la décision de refaire une piqure tout de suite (N120: 349) (Sara).

Concernant l'action de prodiguer les soins requis, Sara se pose des questions. Elle vérifie dans ses notes de références ce qu'elle sait. Elle n'agit que lorsqu'elle est certaine. Dans l'extrait suivant, Sara se remémore les gestes posés dans un milieu d'isolement.

Je ressors (de la chambre), je me déshabille (N110:320). J'apporte seulement ce que j'ai besoin. Après je place tout dans un petit sac (N113: 333) (Sara). 


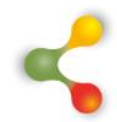

\section{REVUE HYBRIDE DE L'ÉDUCATION}

Cette activité clé comprend également la consultation et l'inscription de notes dans le dossier de la personne soignée. Sara prépare ses interventions en recueillant des renseignements. L'action de compléter les dossiers comprend des éléments relatifs aux pansements à refaire, aux médicaments à donner incluant le soulagement des douleurs avec, par exemple, la prise de narcotique ou encore la pose de blocs salins. Cette action inclut les renseignements à noter lors de l'admission d'une personne. Cette action peut être mise en lien avec les compétences transversales d'ordre social qui occupent une large place dans le Programme de formation de l'école québécoise et qui constituent un levier pour le développement des compétences disciplinaires tout en permettant la distinction entre les experts et les novices (gouvernement du Québec, $2019 b$ ). D'ailleurs, les actions de l'activité clé collaboration avec l'équipe de soins présentées dans la section suivante confirment la coopération et la prise en compte des mutations constantes des environnements de travail (Alexandre, Daigle et Amyot, 2019).

\subsection{Actions de l'activité clé collaboration avec l'équipe de soins}

L'analyse des données montre que les actions liées à l'activité clé collaboration avec l'équipe de soins sont participer à l'attribution des patients entre les membres de l'équipe de soins et soutenir le travail des collègues et des préposés(es). L'action participer à l'attribution des patients entre les membres de l'équipe de soins correspond à la prise en charge d'un certain nombre de patients. Sara mentionne la reconnaissance au sein de l'équipe soignante de cas «lourds ou extrêmes ». Ainsi la répartition des " cas lourds » entre les membres de l'équipe de soins constitue le critère le plus important. Sara ajoute que l'attribution équitable de la quantité de soins à dispenser en lien avec la condition de la personne soignée évite la surcharge de travail d'un membre de l'équipe :

On sépare (les cas lourds) pour que cela ne soit pas plus lourd pour l'une que pour l'autre (N51-147) (Sara).

Lors de l'action soutenir le travail des collègues et des préposés(es), Sara aide ses collègues quand la situation le permet.

J'aidais quand je pouvais, ce qu'elle (ma collègue) ne pouvait pas faire, je l'aidais (N38-111). Je me suis retrouvée à aller voir les autres patients pendant qu'elle s'occupait plus de ce patient. (N64-179-180) (...) Je l'ai vue aller un peu, alors je m'occupais des autres pendant qu'elle s'occupait de lui (N65182-183) (Sara). 


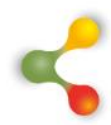

\section{REVUE HYBRIDE DE L'ÉDUCATION}

\subsection{Actions de l'activité clé optimisation de la pratique}

Les résultats obtenus indiquent que l'activité clé optimisation de la pratique se traduit par les actions mettre à jour des connaissances et recourir à une autorité (coordination de l'unité de soins). Concernant l'action mettre à jour des connaissances, Sara mentionne se poser beaucoup de questions et vérifier ses connaissances. Elle s'informe auprès d'une personne qui sait comment «faire» et qui est aussi «facile d'approche ». Elle l'observe.

Concernant l'action recourir à une autorité (coordination de l'unité de soins), Sara fait valoir l'importance de pouvoir se référer à quelqu'un en tout temps.

Je peux me référer en tout temps (N36-105). C'est sûr qu'il a toujours quelqu'un sur chaque département (...) il y a toujours une infirmière (N178-516) (Sara).

\section{Avancées de cette étude}

Dans le sens émis par Hardy et Landreville (1992) sur le savoir professionnel, l'examen des résultats de Sara suggère l'existence d'un processus de raisonnement en Santé, assistance et soins infirmiers. En effet, l'exercice d'un processus de résolution de problèmes semble présent par les actions associées aux quatre activités clés du métier. Les résultats de l'activité clé planification des soins en fonction du milieu semblent confirmer les propos de Pastré (2002) sur le diagnostic d'une situation effectué par les acteurs.

Tout en corroborant les compétences professionnelles identifiées par Roegiers (2010) et l'apprentissage par les collègues souligné par Durand (2005), les résultats laissent également voir des relations entre les activités clés de collaboration avec l'équipe soignante et d'optimisation de la pratique. Ceci met en évidence la coopération et la prise en compte des mutations constantes des environnements de travail. On peut d'ores et déjà rapprocher ces résultats des travaux de Pastré (2016) concernant des manifestations de l'intelligence de la tâche et des dimensions multiples de la résolution de problèmes.

À l'instar de Leplay (2006) sur l'établissement de relation entre un système de représentation d'une situation professionnelle et un système de représentation de l'action (en situation) jumelé à l'expression de la relation par un jugement, l'examen des données de la participante Sara tend à préciser les éléments pris en compte dans le processus décisionnel. 


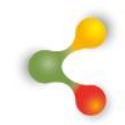

\section{REVUE HYBRIDE DE L'ÉDUCATION}

Lors de la prise d'informations, Sara constate l'état de fatigue du malade et la présence d'un soluté sur l'autre bras. Elle prend la décision de recourir à une autorité, une action de l'activité clé optimisation de la pratique.

L'analyse de l'activité clé application des procédés de soins suggère diverses formes de réalisation autonome du travail au regard de la spécificité du métier. Ces résultats vont dans le sens de l'importance de la compréhension de la nature du savoir de métier mise de l'avant par Alexandre et ses collaboratrices (2016). Quoique partiels, ces résultats semblent prometteurs dans la mesure où l'étude documente les prises de décisions professionnelles de novices avec moins d'un an de pratique sur le marché du travail.

En conclusion, les acteurs de la formation professionnelle, qu'ils soient du corps enseignant, des travailleuses et travailleurs ou des personnes formatrices en milieu de travail, doivent s'adapter aux nouvelles exigences tout en anticipant les changements. Même si, au total, quatre personnes ont participé à l'étude, il est souhaitable que d'autres recherches sur le processus de raisonnement de métier puissent s'ouvrir à un plus grand nombre de personnes participantes exerçant d'autres métiers de la formation professionnelle.

L'explicitation du processus de raisonnement de l'infirmier et de l'infirmière auxiliaires constitue une valeur ajoutée à la compréhension du processus de raisonnement de la personne soignante au cœur d'une situation précise et donne un accès détaillé à la pensée soutenant l'agir professionnel. Ce partage de savoir entre les acteurs du système éducatif et ceux du marché de l'emploi prépare de façon plus efficace l'apprentissage du travail. Les nouvelles avenues générées par cette étude vont permettre de mettre en place des dispositifs de formation adaptés aux défis et enjeux de l'adéquation entre la formation et le marché du travail. 


\section{REVUE HYBRIDE DE L'ÉDUCATION}

\section{Références}

Alexandre, M., Daigle, G. et Amyot, D. (2019). L'agir professionnel et le processus de raisonnement de métier. Éducation Permanente, Horssérie, AFPA, 159-167.

Alexandre, M., Thériault, N. et Daigle G. (2016). Modélisation du savoir professionnel du travailleur du compagnon et de l'enseignant en techniques d'usinage: le processus de raisonnement de métier. Revue Travail et Apprentissages, 18(2), 7-23.

Alexandre, M. (2013). La description du savoir didactique d'enseignantes expérimentées en Techniques d'éducation à l'enfance en situation de planification, d'intervention et de réflexion : trois études de cas [thèse de doctorat, Université de Sherbrooke]. Savoirs. https://savoirs.usherbrooke.ca/handle/11143/6378

Benner, P. (1995). De novice à expert: excellence en soins infirmiers. ERPI.

Association de didactique professionnelle (2018). Former et développer l'intelligence professionnelle. Appel à communications. https://fourwaves-

sots.s3.amazonaws.com/static/media/attached/1190/Colloque RP DP2019-Appel-a-comm.pdf

Direction des soins infirmiers et direction des ressources humaines. (2009). Programme de soutien clinique à la pratique des infirmières et infirmières auxiliaires. Hôpital Louis-H. Lafontaine.

Durand, C. (2005) De novice à expert: le processus de professionnalisation (première partie). Cadre de santé. http:// www.Cadredesante.com/spip/profession/pedagogie.

Gouvernement du Québec. (2019a). Exploration de la formation professionnelle. Ministère de l'éducation et de l'enseignement supérieur.

http://www.education.gouv.qc.ca/fileadmin/site web/documents/ed ucation/jeunes/pfeg/PFEQ exploration-formationprofessionnelle.pdf

Gouvernement du Québec. (2019b). Loi médicale. http://legisquebec.gouv.qc.ca/fr/ShowDoc/cs/M-9

Gouvernement du Québec. Règlements et autres actes. Gazette Officielle du Québec, (19). 


\section{REVUE HYBRIDE DE L'ÉDUCATION}

Fédération Interprofessionnelle de la Santé du Québec. (2008). Rôles, tâches, fonctions et responsabilités des professionnelles en soins: quel est le portrait en 2008 ? Document public d'information présenté au conseil fédéral mars 2008.

Fédération Interprofessionnelle de la Santé du Québec (2009). Révision du système professionnel: Les impacts de la Loi 90. http://www.figsante.qc.ca/publicfiles/.../ot 0903 impacts-de-la-loi$\underline{90 \text { fr.pdf }}$

Hardy, M. et Landreville, A. (1992). Pour une analyse des processus d'appropriation du savoir professionnel. Revue des sciences de l'éducation, 18(3), 463-473. http ://id.erudit.org/iderudit/900748ar, doi : 10.7202/900748ar

Hould, G. (2011). Intégration des infirmières auxiliaires au CHU SainteJustine. $\quad$ https://www.chusj.org/...pdf actualites pratiquesexemplaires integration-infirmieres-auxiliaires.pdf.aspx

Karsenti, T. et Demers, S. (2011). L'étude de cas. Dans T. Karsenti et L. Savoie-Zajc (dir.), La recherche en éducation : étapes et approches (3e éd.) (p. 229-252). ERPI.

Lechasseur, K. (2009). Mobilisation des savoirs pour une pensée critique chez des étudiantes infirmières bachelières en situation de soin [thèse de doctorat inédite]. Université Laval.

Leplat, J. (2000). L'analyse psychologique de l'activité en ergonomie. Octares.

Leplay, E. (2006). Co-construction de savoirs professionnels par la recherche : vers un dispositif expérimental en formation initiale de travailleurs sociaux. Esprit critique, 8(1).

Ministère de l'Éducation. (2000). La formation professionnelle et technique. Santé, assistance et soins infirmiers. Programme d'études 5235. Gouvernement du Québec.

Ministère de l'Éducation. (2009). La formation professionnelle et technique. Santé, assistance et soins infirmiers. Programme d'études 5325. Gouvernement du Québec.

Ordre des Infirmières et Infirmiers Auxiliaires du Québec. (2011). Les activités professionnelles de l'infirmière auxiliaire: champ d'exercice, activités réservées et autorisées. OIIAQ. 


\section{REVUE HYBRIDE DE L'ÉDUCATION}

Paillé, P. et Mucchielli, A. (2003). L 'analyse qualitative en sciences humaines et sociales. Armand Colin.

Pastré, P. (2002). L'analyse du travail en didactique professionnelle. Revue française de pédagogie, 138, 9-17.

Pastré, P. (2016). Les compétences professionnelles et leur développement. La revue de la CFDT. http//:benhur.teluq.uquebec.ca/SPIP/inf4018/IMG/pdf/Pastre.pdf

Perrenoud, P. (1994). L'ambiguïté des savoirs et du rapport au savoir dans le métier d'enseignant. http://www.unige.ch/fapse/SSE/teatchers/perrenoud/php main/php 1994/1994 03.html

Perrenoud, P. (2004). Adosser la pratique réflexive aux sciences sociales de l'éducation. Dans J. - F. Inisan, (dir.), Analyse de pratiques et attitude réflexive en formation (p. 11-32). Reims.

Roegiers X. (2012). Quelles réformes pédagogiques pour l'enseignement supérieur? De Boeck Supérieur.

Roegiers, X. (2010). Des curricula pour la formation professionnelle initiale : la pédagogie de l'intégration comme cadre de réflexion et d'action pour l'enseignement technique et professionnel. De Boeck Université.

Roy, S. (2009). L'Étude de cas. Dans B. Gauthier (dir.), Recherche sociale : De la problématique à la collecte de données (5éd.) (p.199-225). Presses de l'Université du Québec.

Vermersch, P. (2014). L'entretien d'explicitation. ESF.

Vermersch, P. (2012). Explicitation et phénoménologie. Presses universitaires de France. 